\title{
Autozygome and high throughput confirmation of disease genes candidacy
}

\author{
Sateesh Maddirevula, $\mathrm{PhD}^{1}$, Fatema Alzahrani, BS ${ }^{1}$, Mohammed Al-Owain, $\mathrm{MD}^{2,3}$, \\ Mohammad A. Al Muhaizea, MD ${ }^{3,4}$, Husam R. Kayyali, MD ${ }^{5}$, Amal AlHashem, MD ${ }^{6}$, \\ Zuhair Rahbeeni, MD², Maha Al-Otaibi, MD, Hamad I. Alzaidan, MD²,3 Ameera Balobaid, MS $^{2}$, \\ Heba Y. El Khashab, MD ${ }^{8,9}$, Dalal K. Bubshait, MD ${ }^{10}$, Maha Faden, MD ${ }^{7}$, Suad Al Yamani, MD ${ }^{4}$, \\ Omar Dabbagh, MD ${ }^{4}$, Mariam Al-Mureikhi, MD ${ }^{11}$, Abdulla Al Jasser, MD ${ }^{6}$, Hessa S. Alsaif, BS ${ }^{1}$, \\ Iram Alluhaydan, MD ${ }^{12}$, Mohammed Zain Seidahmed, MD ${ }^{13}$, Bashair Hamza Alabbasi, MD ${ }^{6}$, \\ Ibrahim Almogarri, MD ${ }^{14}$, Wesam Kurdi, MD ${ }^{15,16}$, Hana Akleh, MD ${ }^{15}$, Alya Qari, MD², \\ Saeed M. Al Tala, MD ${ }^{17}$, Suzan Alhomaidi, MD ${ }^{7}$, Amal Y. Kentab, MD ${ }^{18}$, Mustafa A. Salih, MD ${ }^{18}$, \\ Aziza Chedrawi, MD ${ }^{4}$, Seham Alameer, MD ${ }^{19}$, Brahim Tabarki, MD ${ }^{6}$, Hanan E. Shamseldin, MVsc ${ }^{1}$, \\ Nisha Patel, PhD ${ }^{1}$, Niema Ibrahim, MS ${ }^{1}$, Firdous Abdulwahab, BS ${ }^{1}$, Menasria Samira, MS ${ }^{1}$, \\ Ewa Goljan, MS ${ }^{1}$, Mohamed Abouelhoda, $\mathrm{PhD}^{1,20}$, Brian F. Meyer, PhD ${ }^{1,20}$, Mais Hashem, BS ${ }^{1}$, \\ Ranad Shaheen, $\mathrm{PhD}^{1}$, Saad AIShahwan, $\mathrm{MD}^{6}$, Majid Alfadhel, MD ${ }^{21}$, Tawfeg Ben-Omran, MD ${ }^{11}$, \\ Mohammad M. Al-Qattan, MD ${ }^{22}$, Dorota Monies, PhD ${ }^{1,20}$ and Fowzan S. Alkuraya, MD ${ }^{1,3,6,20}$
}

Purpose: Establishing links between Mendelian phenotypes and genes enables the proper interpretation of variants therein. Autozygome, a rich source of homozygous variants, has been successfully utilized for the high throughput identification of novel autosomal recessive disease genes. Here, we highlight the utility of the autozygome for the high throughput confirmation of previously published tentative links to diseases.

Methods: Autozygome and exome analysis of patients with suspected Mendelian phenotypes. All variants were classified according to the American College of Medical Genetics and Genomics guidelines.

Results: We highlight 30 published candidate genes (ACTL6B, ADAM22, AGTPBP1, APC, C12orf4, C3orf17 (NEPRO), CENPF, CNPY3, COL27A1, DMBX1, FUT8, GOLGA2, KIAA0556, LENG8, MCIDAS, MTMR9, MYH11, QRSL1, RUBCN, SLC25A42, SLC9A1, TBXT, TFG, THUMPD1, TRAF3IP2, UFC1, UFM1, WDR81, $X R C C 2, Z A K)$ in which we identified homozygous likely deleterious variants in patients with compatible phenotypes. We also identified homozygous likely deleterious variants in 18 published candidate genes (ABCA2, ARL6IP1, ATP8A2, CDK9, CNKSR1, DGAT1, DMXL2, GEMIN4, HCN2, HCRT, MYO9A, PARS2, PLOD3, PREPL, SCLT1, STX3, TXNRD2, WIPI2) although the associated phenotypes are sufficiently different from the original reports that they represent phenotypic expansion or potentially distinct allelic disorders.

Conclusions: Our results should facilitate the timely relabeling of these candidate disease genes in relevant databases to improve the yield of clinical genomic sequencing.

Genetics in Medicine (2019) 21:736-742; https://doi.org/10.1038/s41436018-0138-x

Key words: ACMG guidelines; variant interpretation; candidate genes

\section{INTRODUCTION}

Mendelian genetics has seen some of the most successful applications of genomic medicine. The often-clear departure of Mendelian phenotypes from the population average, the high confidence with which these phenotypes are attributed to single variants, and the rare and deleterious nature that often characterizes these variants are important contributing factors. ${ }^{1}$ A major concern in Mendelian genetics, however, is that there are potentially thousands of genes that have yet to be linked to Mendelian phenotypes, a critical gap that greatly impedes the full realization of the benefits of clinical genomic sequencing. For example, the commonly cited 25\% clinical sensitivity of genomic sequencing in the setting of Mendelian diseases appears to correlate with the extent to which the genetic heterogeneity of a particular phenotype had been captured by prior research. ${ }^{2}$ Similarly, recent

Correspondence: Fowzan S. Alkuraya (falkuraya@kfshrc.edu.sa). "Affiliations are listed at the end of the paper. 
papers suggest that revisiting exome sequencing based on newly annotated disease genes can boost the yield. ${ }^{3-7}$ Thus, it remains a priority in the field of clinical laboratory genetics to have all genes with links to Mendelian phenotypes established in databases that are commonly used in the variant annotation pipeline, e.g., OMIM. The American College of Medical Genetics and Genomics (ACMG) guidelines for variant interpretation clearly state that variants that would otherwise qualify as likely pathogenic or even pathogenic cannot be reported as such in genes with no established link to disease, and as such these variants should be reported, if at all, as variants of unknown significance. ${ }^{8}$

The International Rare Diseases Research Consortium (IRDiRC) states in its vision that all patients with genetic disorders should receive a molecular diagnosis by the year 2020 (ref. ${ }^{9}$ ). It is more realistic, however, to hope that the entire Mendeliome will be curated by that date. This optimism is fueled by the remarkable rate at which novel disease genes are identified, thanks in large part to the democratization of genomic sequencing that made it possible for the first time for patients to have their genomes sequenced clinically thus bypassing the bottleneck of testing by research labs with specific interest in their phenotypes. ${ }^{10}$ Notwithstanding the concern that the pace of such discoveries may have slowed, a new bottleneck has quickly emerged in that as we tackle diseases of exceeding rarity, we are more likely to face the " $n$ of one" challenge. ${ }^{11}$ While publishing candidate genes based on single pathogenic variants is often insufficient to establish links to diseases unambiguously, such publications are often the impetus for follow-up confirmatory papers. The percentage of novel disease genes that are subsequently confirmed when their initially published candidacy was based on single mutational event is unknown. However, this percentage likely depends on the strength of other lines of supporting evidence in the original publication, e.g., positional mapping, segregation, functional analysis, etc. For example, of the 66 candidate genes we published based on single pathogenic variants that are also supported by compelling positional mapping data, i.e., a single autozygous locus, 54 were subsequently confirmed and none was challenged or disputed (unpublished data).

The power of autozygosity, however, is not limited to the initial mapping and reporting of novel disease genes. As a vast source of homozygous variants, the autozygome may also serve as an efficient tool for the high throughput confirmation of disease genes candidacy by supplying additional deleterious variants in those gene. Even when the same previously reported variants are encountered in "unrelated" patients with the same haplotypes, there is further strengthening of the previously published positional mapping data and thus the candidacy of the respective genes. In this study, the largest of its kind, we implemented this approach to support the candidacy of 48 previously reported novel candidate disease genes.

\section{MATERIALS AND METHODS}

\section{Human subjects}

Subjects were either consented as part of an institutional review board-approved research protocol (in the case of research-grade exomes, KFSHR RAC 2121053, 2140016, 2080006,2070023 ) or a standard clinical exome consent (in the case of clinical exomes). Phenotypes were provided by indepth clinical evaluation by the participating clinicians. Where applicable, additional consent to show identifiable patient information were also obtained.

\section{Variant identification}

We searched our database of 799 research and 1750 clinical exomes in search of homozygous variants that otherwise qualify for pathogenic or likely pathogenic classification according to the ACMG guidelines but could not be classified as such because the involved genes only have tentative links to diseases. We defined genes as having tentative links if they belonged to one of the following three categories:

a. genes with previously reported pathogenic variants in the context of a Mendelian phenotype but remain unlisted as disease genes in OMIM

b. genes that are listed in OMIM as disease genes but with "?" mark indicating insufficient evidence

c. genes that are listed in OMIM as diseases genes without "?" mark but the evidence is based on a single study

The autozygome was defined by the entire set of autozygous blocks per individual. ${ }^{12}$ Determining whether the variant resides within the autozygome was made directly from the exome data as described in detail elsewhere. ${ }^{13}$

\section{Potential confirmation and phenotypic definition}

We considered two categories for the potential confirmation of candidacy. Category 1 is when a homozygous variant other than the one previously reported is identified. Category 2 is when the previously reported candidacy was based on a single homozygous variant and we identified the same variant on the same haplotype block in an individual who is not part of the originally reported sibship. For defining the phenotypic overlap, we considered three classes:

Class 1: The phenotype is identical or very similar to that previously reported.

Class 2: The phenotype is similar but involves additional features, i.e., phenotypic expansion.

Class 3: The phenotype was sufficiently different to qualify as a potentially different clinical presentation, i.e., distinct allelic disorder. We opted to consider this possibility rather than challenge or dispute the previous reports to accommodate the increasing appreciation of variable phenotypic expression of Mendelian genes. ${ }^{14}$

\section{RESULTS}

\section{Potential confirmation of $\mathbf{4 8}$ previously reported novel} disease genes

Supplementary Table 1 summarizes the 52 cases (44 reported here for the first time and their full clinical phenotypes are 
described in Table S1) whose autozygome contained homozygous variants that argue in support of previously reported tentative disease-gene links. These variants were mostly $(n=$ $32, \sim 66 \%)$ truncating thus potentially rendering the affected individuals natural knockouts for the respective genes, and as such confer further confidence in the previously reported links (especially when such links were based on hypomorphic missense variants). ${ }^{15}$ Examples include $A B C A 2, H C N 2$, MYO9A, QRSL1, and THUMPD1. Missense variants were similarly carefully chosen to be likely deleterious based on novelty or extreme rarity (minor allele frequency $[\mathrm{MAF}]<$ 0.001 in gnomAD and local exome database), and consistent deleterious in silico prediction by SIFT, PolyPhen, and CADD (Supplementary Table 1).

The overwhelming majority of confirmation events were based on category 1 , i.e., independent pathogenic variant events. We only encountered five instances of category 2 , i.e., same previously reported variant on a similar haplotype, and these involved CDK9, GEMIN4, C3orf17, WDR81, SCLT1, and UFC1. We have previously reported a single pathogenic variant each in CDK9, GEMIN4, C3orf17, WDR81, SCLT1, and UFC1 in patients with CHARGE-like presentation (coloboma, choanal atresia, and urogenital malformations), ${ }^{16}$ syndromic cataract (cataract with static encephalopathy), ${ }^{17}$ Ehlers-Danlos-like syndrome, ${ }^{16,18}$ congenital hydrocephalus (another variant in WDR81 was also reported), ${ }^{19}$ oral-facial-digital syndrome (OFD), ${ }^{20}$ and epileptic encephalopathy, ${ }^{13}$ respectively. We have identified the same founder variants in individuals with the same phenotypes that we previously attributed to these genes. In addition, it is worth highlighting that in two instances, we report recessive pathogenic variants in genes previously proposed as candidate genes based on monoallelic pathogenic variants: HCRT and WIPI2.

\section{Defining the phenotypic spectrum of previously reported candidate genes}

We have encountered 30 genes with mutational events that represent no clear phenotypic expansion, i.e., class 1 (Supplementary Table 1 and Fig. 1). We have also encountered 17 instances (15 genes) of phenotypic expansion, i.e., class 2 (Supplementary Table 1 and Fig. 2). A few of these are worth highlighting. For example, the tentative OMIM phenotype for ARL6IP1 is spastic paraplegia based on a single homozygous frameshift variant. However, the patient in whom we identified a homozygous truncating variant presented with severe neonatal hypotonia. Similarly, the only reported patient with the OMIM phenotype of cerebellar ataxia, mental retardation, and dysequilibrium syndrome had a single missense variant in ATP8A2 whereas the patient we present with a homozygous truncating variant has physical and cognitive delay, failure to thrive, vision impairment, hypotonia, eczema and leukodystrophy. Another interesting example is lysyl hydroxylase 3 deficiency, which is listed in OMIM based on a single compound heterozygous pathogenic variant. While our patient with a homozygous truncating variant similarly has significant connective tissue involvement, his presentation seems to expand the phenotype by featuring microcephaly, and ptosis (Fig. 2). Similarly, our patient 17-3959 with a homozygous truncating variant in TXNRD2 had a syndromic manifestation characterized by low cortisol, intellectual disability, epilepsy, dysmorphic features, truncus arteriosus, and omphalocele (Fig. 2), unlike isolated glucocorticoid deficiency that was reported in OMIM. Case 17DG1005 is rather unusual in that it represents a phenotypic "contraction" rather than expansion. In 2015, we reported the identification of SCLT1 along with TBC1D32/C6orf170 as two novel genes for OFD IX based on two families with severe midline defects including cleft and hypopituitarism, in addition to microphthalmia and polydactyly. ${ }^{20}$ The same SCLT1 founder mutation was later identified in a child with a milder form of intellectual disability, obesity, coloboma, renal failure, and subsequent renal transplant, retinopathy, midline cleft, epilepsy, atopy, panhypopituitarism, and undescended left testicle. ${ }^{21}$ However, case 17DG1005 and his sibling both presented with isolated panhypopituitarism and hypothalamic hamartoma with no associated syndromic features of OFD or other ciliopathies (Fig. 2). PARS2 was previously implicated in a mitochondrial phenotype featuring neurodegeneration and liver involvement (Alpers syndrome) based on compound heterozygous pathogenic variants. ${ }^{22-24}$ Interestingly, in one family we show that homozygosity for a variant in PARS2 (NM_152268.3:c.283G>A:p.[Val95Ile]), previously reported only in trans with another allele, fully segregated with global developmental delay, epilepsy, and brain atrophy but without lactic acidosis or renal or liver involvement (Fig. 2).

In class 3 , the phenotypes observed are sufficiently different from those previously reported that we argue these may represent distinct allelic disorders (Supplementary Table 1, Fig. 2). These include two families with global developmental delay and intellectual disability (Fig. 2) that fully segregated with homozygous truncating variants in ABCA2 (NM 212533.2:c.740dupT:p.[Gly248Argfs $\left.{ }^{\star} 38\right]$ ) and (NM_212533. 2:c.1027C $>$ T:p. [Gln343*]), which is distinct from the amyotrophic lateral sclerosis reported by Steinberg et al. ${ }^{25} \mathrm{We}$ also found that DMXL2 harbors a homozygous truncating variant (NM_001174117.1:c.4349_4350insTTACATGA:p.[Glu1450A spfs $\left.^{\star} 23\right]$ ) in a child with intellectual disability, epilepsy, macrocephaly, and dysmorphism (Fig. 2), in contrast to the multiple endocrinopathies reported by Tata et al. based on a single pathogenic variant. ${ }^{26}$ Finally, WIPI2 was reported as a candidate gene for cerebral palsy based on a single de novo pathogenic variant by McMichael et al. ${ }^{27}$ However, our patient with a homozygous truncating variant in this gene presented with hypotonia, failure to thrive, nystagmus, elevated transaminases, and raised $\alpha$-fetoprotein.

\section{DISCUSSION}

The growing demand for clinical sequencing calls for an acceleration in curating the Mendeliome. Variants in the Mendeliome are not only helpful in diagnosis and management, but also in reproductive planning and even in 

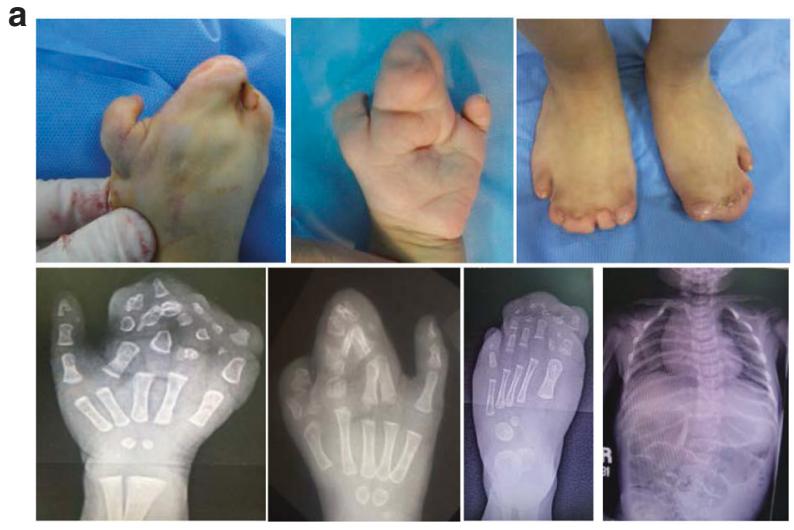

APC b
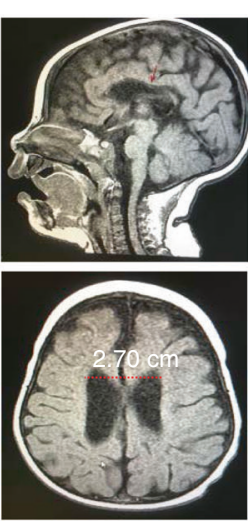

ACTL6B
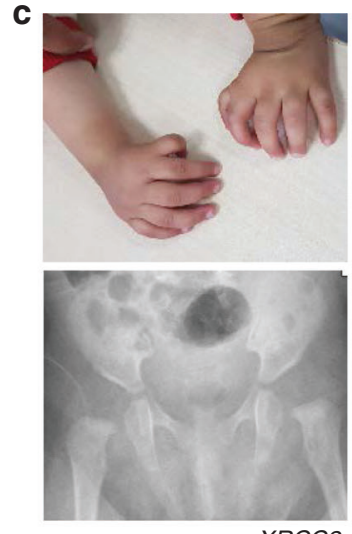

$X R C C 2$
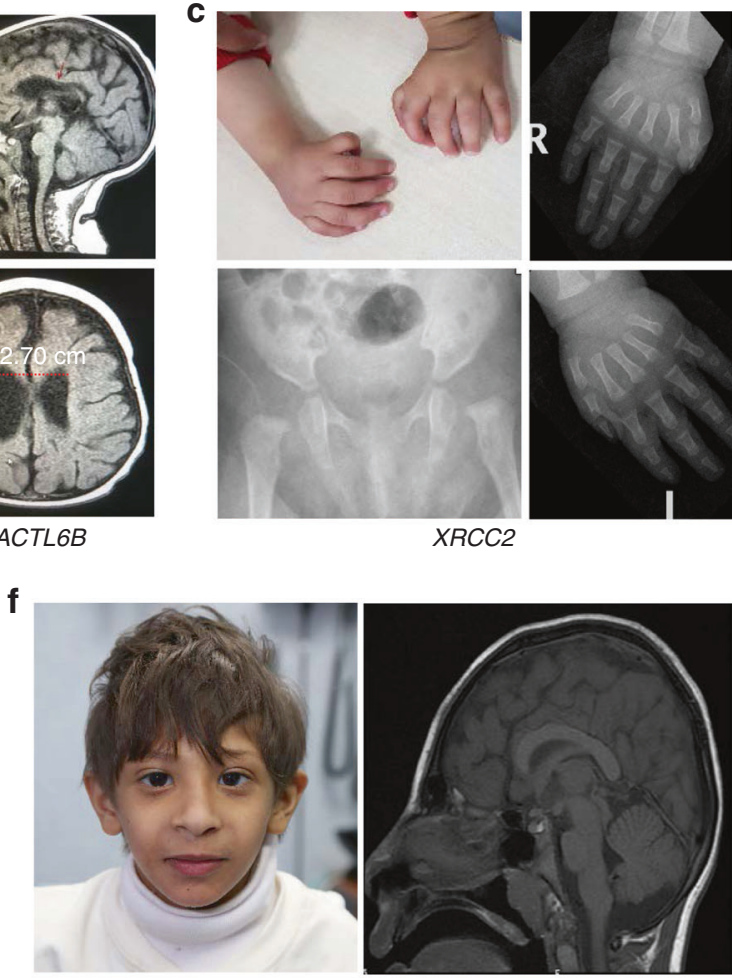

CENPF

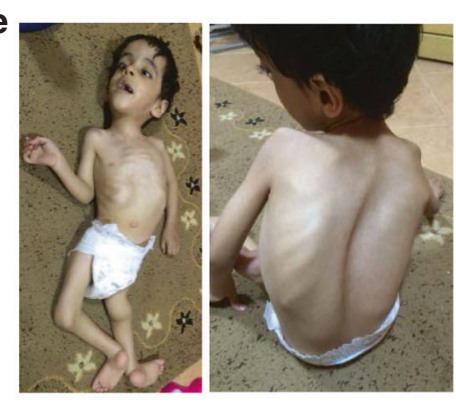

COL27A1

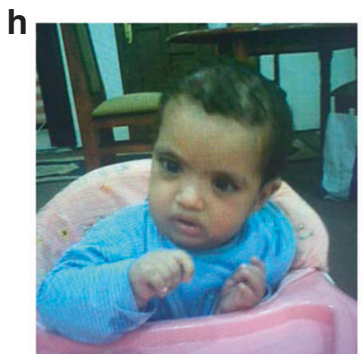

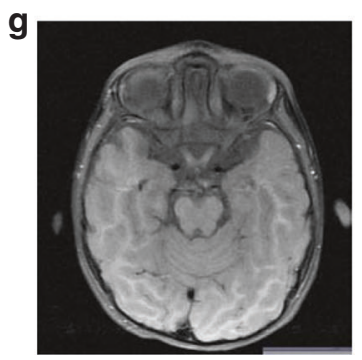

KIAA0556

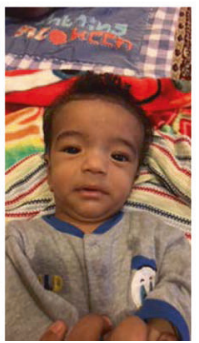

FUT8

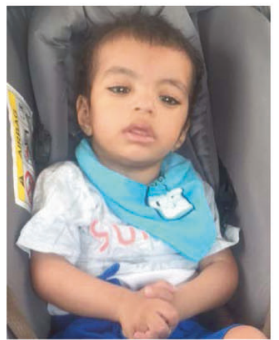

(1)

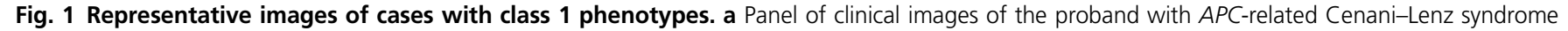
showing the classic bony syndactyly with loss of the normal configuration of the phalanges. b Brain magnetic resonance image (MRI) of the proband with pathogenic variant in ACTL6B showing agenesis of corpus callosum and mild ventricular dilatation (2.70 cm). c Typical skeletal findings in the proband with XRCC2-related Fanconi anemia showing hypoplastic thumbs and dysplastic hips. $\mathbf{d}$ Brain MRI image showing thin but complete corpus callosum in the proband with GOLGA2 pathogenic variant. e Clinical images of the proband with Steel syndrome (pathogenic variant in COL27A1) showing characteristic facial and skeletal appearance (elongated face with severe scoliosis). $\mathbf{f}$ Facial photograph and MRI brain of the proband with pathogenic variant in CENPF showing small head, relatively large ears, epicanthal folds, blue-tinged sclerae, prominent nose, thin upper lip, and small chin. MRI revealed reduced white matter volume and marginal pachygyria. $\mathbf{g}$ Brain MRI image showing mild molar tooth sign in the proband with KIAA0556 pathogenic variant. $\mathbf{h}$ Facial photographs of proband with FUT8 pathogenic variant showing coarse face with synophrys, hypertelorism, sparse eye-lashes, low set ears, thick inverted V-shaped lips.

identifying drug targets for common diseases. ${ }^{1,28,29}$ It is likely that in the near future, the overwhelming majority of patients with Mendelian diseases will have their causal variants captured by genome sequencing. However, the confidence with which such variants are called as such will depend on whether the genes in which these variants are identified had been linked to the patient's phenotype. Sharing of variants across different cohorts will likely accelerate successful matching. ${ }^{30}$ However, it is likely that postpublication matchmaking will remain an important method for the independent validation of candidate genes. In this study, we attempted to maximize the power of postpublication matchmaking by reporting a large group of likely deleterious homozygous variants that were facilitated by the phenomenon of autozygosity in genes previously published as candidates. While there is value in dedicating a separate publication to each of these confirmatory reports, we believe that their grouping will provide a more timely communication of results.

Not surprisingly, the phenotypes we report in each of the confirmatory cases range from identical to quite distinct from those previously reported. We have previously shown how 

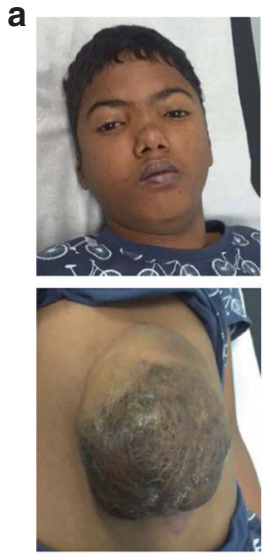

TXNRD2
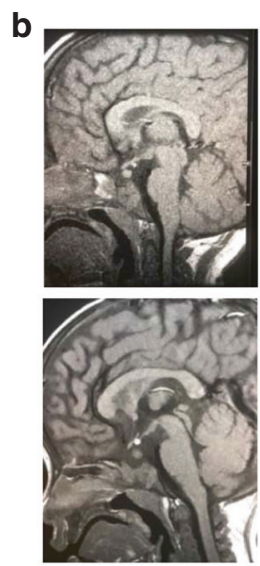

SCLT1
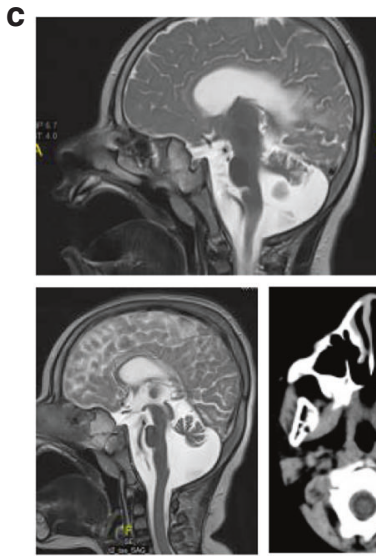

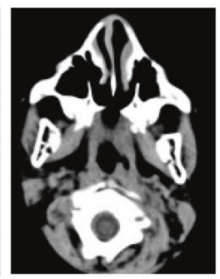

CDK9
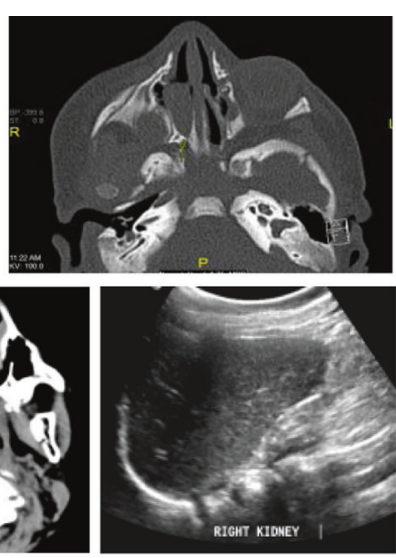

f
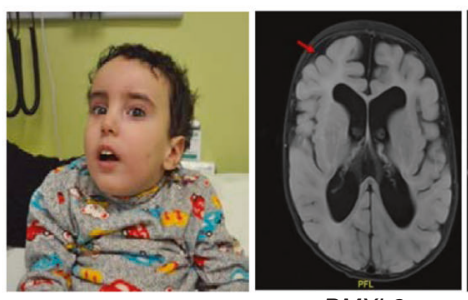

$D M X L 2$
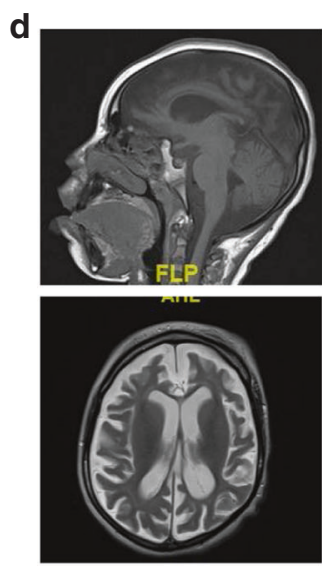

PARS2

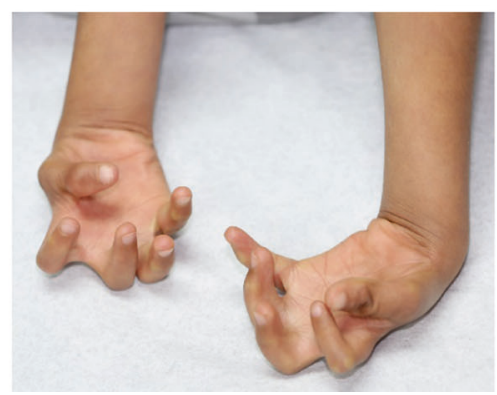

PLOD3

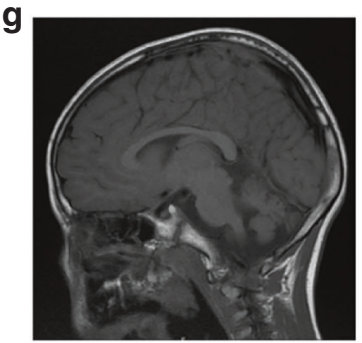

GEMIN4 h

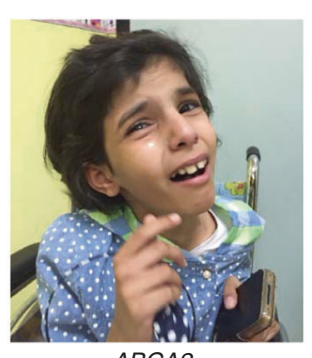

Fig. 2 Representative images of cases with class 2 and 3 phenotypes. a Clinical images of the proband with TXNRD2 pathogenic variant showing dysmorphic facies and a large umbilical hernia. b The two siblings with SCLT1 pathogenic variant showing absence of the anterior lobe of pituitary gland with the evidence of ectopia of the posterior pituitary gland and thalamic hamartoma. c Three families are with CDK9-related CHARGE-like phenotype showing cerebellar atrophy, unilateral choanal atresia, and dysplastic atrophic kidney. $\mathbf{d}$ Brain magnetic resonance image (MRI) of proband with pathogenic variant in PARS2 showing severe cerebral volume loss indicating severe brain atrophy, thinning of the corpus callosum, and severe cerebral volume loss in the supratentorial and infratentorial area. e Clinical images of the proband with PLOD3 pathogenic variant showing dysmorphic facies (prominent forehead, short and flat nose, ptosis with compensatory arching of eyebrows, posteriorly rotated low set ears) and hand contractures. $\mathbf{f}$ Facial features and MRI images of proband with $D M X L 2$ pathogenic variant represent long face, high forehead, short philtrum, low set ears, and moderate degree of cerebral and brainstem atrophy. $\mathbf{g}$ Brain MRI image showing small atrophic cerebellum with prominence of the posterior fossa cerebrospinal fluid (CSF) spaces in the proband with GEMIN4 pathogenic variant. $\mathbf{h}$ Facial pictures of index (16DG0071) subject with ABCA2 showing apparent lack of gross dysmorphism.

recessive human knockouts can present with surprisingly different phenotypes compared with those with monoallelic pathogenic variants, and this study adds several examples of this phenomenon, e.g., MYH11-related megacystis. ${ }^{14}$ However, we also note that in several other cases, marked phenotypic heterogeneity was noted even when compared with previously reported cases with biallelic pathogenic variants. Although we have looked carefully for additional variants that may have resulted in these unusual phenotypes (i.e., multilocus phenotypes, also referred to as dual molecular diagnosis), none were identified in the cases presented. Thus, it seems likely that these cases may represent true phenotypic expansion, especially considering the very limited cases that had been reported. Obviously, additional cases in the future will be needed to delineate the true phenotypic spectrum of the respective genes.

The ClinGen consortium has recently published an attempt to standardize the establishment of disease-gene links. ${ }^{31}$ This will likely be one of many steps going forward as we grapple with such issues as extreme phenotypic heterogeneity and exceedingly rare diseases. The uncertainty that surrounds many disease-gene links can be seen in OMIM disease genes listings, which sometimes include genes with single spathogenic variants but not genes with several reported pathogenic variants. While it is agreed that two independent pathogenic variants are not necessarily sufficient to unambiguously link a 
gene to a specific phenotype, this remains one of the best confirmatory methods. On the other hand, compelling positional mapping data may sometimes be the only possible means of confirmation especially for phenotypes that are allele-specific. For example, although only one single missense variant in ADAT3 has been reported, it has emerged as the single most common cause of nonsyndromic autosomal recessive intellectual disability in Arabia such that there is now little doubt about the disease-gene link..$^{32-35}$ It is for this reason that we opted to include in this study, as another confirmatory method, cases with similar variants to those previously reported if they corroborate the positional mapping evidence even as we acknowledge the possibility of being in linkage disequilibrium with the actual causal variants. Examples of disorders we were able to confirm through this approach in this study are TBXT-related myelomeningocele, ${ }^{36}$ $A P C$-related Cenani-Lenz syndrome, ${ }^{37}$ and XRCC2-related Fanconi anemia. ${ }^{38}$

There is now a growing appreciation that deleterious recessive variants may express phenotypically in different ways depending on whether they are encountered in homozygosity or in the compound heterozygous state with other variants. Thus, the autozygome provides a unique opportunity to unmask the phenotypic expression of variants that had only been reported as compound heterozygous. ${ }^{39}$ For example, we show here that one PARS2 allele that was reported to cause Alpers syndrome when compound heterozygous only causes nonspecific global developmental delay and brain atrophy when homozygous but with no evidence of lactic acidosis or liver involvement when homozygous. With its potential to render many previously reported variants as homozygous, the autozygome can be very powerful in revealing important phenotypic aspects of alleles, which may not be appreciated otherwise.

In summary, we present the largest confirmatory cohort to date in the setting of genes with tentative links to Mendelian phenotypes, all based on autozygome-related homozygosity for likely deleterious variants. This effort will complement others to accelerate the high confidence mapping of the Mendeliome. The resulting phenotypes, whether overlapping or distinct, will add to our understanding of the medical relevance of the respective genes in different contexts, and will further enhance the accuracy of variant interpretation.

\section{ELECTRONIC SUPPLEMENTARY MATERIAL}

The online version of this article (https://doi.org/10.1038/s41436$018-0138-x)$ contains supplementary material, which is available to authorized users.

\section{ACKNOWLEDGEMENTS}

We thank the study patients for their enthusiastic participation. We thank the Sequencing and Genotyping Core Facilities at King Faisal Specialist Hospital \& Research Center (KFSRHC) for their technical help. This work was supported in part by King Salman Center for Disability Research, King Abdulaziz City for Science and Technology and the Saudi Human Genome Program.

\section{DISCLOSURE}

The authors declare no conflicts of interest.

\section{REFERENCES}

1. Alkuraya FS. Discovery of mutations for Mendelian disorders. Hum Genet. 2016;135:615-23.

2. Wright $C F$, FitzPatrick DR, Firth HV. Paediatric genomics: diagnosing rare disease in children. Nat Rev Genet. 2018;19:253-68.

3. Wright CF, McRae JF, Clayton $S$, et al. Making new genetic diagnoses with old data: iterative reanalysis and reporting from genome-wide data in 1,133 families with developmental disorders. Genet Med. 2018 January 11; https://doi.org/10.1038/gim.2017.246. [Epub ahead of print].

4. Nambot $S$, Thevenon J, Kuentz $P$, et al. Clinical whole-exome sequencing for the diagnosis of rare disorders with congenital anomalies and/or intellectual disability: substantial interest of prospective annual reanalysis. Genet Med. 2017;20:645-54.

5. Eldomery MK, Coban-Akdemir Z, Harel T, et al. Lessons learned from additional research analyses of unsolved clinical exome cases. Genome Med. 2017;9:26.

6. Shamseldin HE, Maddirevula S, Faqeih E, et al. Increasing the sensitivity of clinical exome sequencing through improved filtration strategy. Genet Med. 2017;19:593.

7. Smith ED, Radtke $K$, Rossi $M$, et al. Classification of genes: standardized clinical validity assessment of gene-disease associations aids diagnostic exome analysis and reclassifications. Hum Mutat. 2017; 38:600-8.

8. Richards S, Aziz N, Bale S, et al. Standards and guidelines for the interpretation of sequence variants: a joint consensus recommendation of the American College of Medical Genetics and Genomics and the Association for Molecular Pathology. Genet Med. 2015;17:405.

9. Austin CP, Cutillo CM, Lau LP, et al. Future of rare diseases research 2017-2027: an IRDiRC perspective. Clin Trans/ Sci. 2017;11:21-27.

10. Dawkins HJ, Draghia-Akli R, Lasko $P$, et al. Progress in rare diseases research 2010-2016: an IRDiRC perspective. Clin Transl Sci. 2017;11: $11-20$.

11. Boycott KM, Rath A, Chong JX, et al. International cooperation to enable the diagnosis of all rare genetic diseases. Am J Hum Genet. 2017;100: 695-705.

12. Alkuraya FS. Autozygome decoded. Genet Med. 2010;12:765.

13. Monies D, Abouelhoda M, AlSayed M, et al. The landscape of genetic diseases in Saudi Arabia based on the first 1000 diagnostic panels and exomes. Hum Genet. 2017;136:921-39.

14. Monies D, Maddirevula S, Kurdi W, et al. Autozygosity reveals recessive mutations and novel mechanisms in dominant genes: implications in variant interpretation. Genet Med. 2017;19:1144.

15. Alkuraya FS. Human knockout research: new horizons and opportunities. Trends Genet. 2015;31:108-15.

16. Shaheen R, Patel N, Shamseldin H, et al. Accelerating matchmaking of novel dysmorphology syndromes through clinical and genomic characterization of a large cohort. Genet Med. 2016;18:686.

17. Alazami AM, Patel N, Shamseldin HE, et al. Accelerating novel candidate gene discovery in neurogenetic disorders via whole-exome sequencing of prescreened multiplex consanguineous families. Cell Rep. 2015;10: 148-61.

18. Maddirevula S, Alsahli S, Alhabeeb L, et al. Expanding the phenome and variome of skeletal dysplasia. Genet Med. 2018 April 5; https://doi.org/ 10.1038/gim.2018.50. [Epub ahead of print].

19. Shaheen R, Sebai MA, Patel N, et al. The genetic landscape of familial congenital hydrocephalus. Ann Neurol. 2017;81:890-7.

20. Adly N, Alhashem A, Ammari A, Alkuraya FS. Ciliary genes TBC1D32/ C6orf170 and SCLT1 are mutated in patients with OFD type IX. Hum Mutat. 2014;35:36-40.

21. Shaheen R, Szymanska K, Basu B, et al. Characterizing the morbid genome of ciliopathies. Genome Biol. 2016;17:242.

22. Mizuguchi T, Nakashima M, Kato M, et al. PARS2 and NARS2 mutations in infantile-onset neurodegenerative disorder. J Hum Genet. 2017;62: 525

23. Pronicka E, Piekutowska-Abramczuk D, Ciara E, et al. New perspective in diagnostics of mitochondrial disorders: two years' experience with whole-exome sequencing at a national paediatric centre. J Trans/ Med. $2016 ; 14: 174$ 
24. Sofou K, Kollberg G, Holmström M, et al. Whole exome sequencing reveals mutations in NARS2 and PARS2, encoding the mitochondrial asparaginyl-tRNA synthetase and prolyl-tRNA synthetase, in patients with Alpers syndrome. Mol Genet Genomic Med. 2015;3:59-68.

25. Steinberg KM, Yu B, Koboldt DC, Mardis ER, Pamphlett R. Exome sequencing of case-unaffected-parents trios reveals recessive and de novo genetic variants in sporadic ALS. Sci Rep. 2015;5:9124.

26. Tata $B$, Huijbregts $L$, Jacquier $S$, et al. Haploinsufficiency of $D m x / 2$, encoding a synaptic protein, causes infertility associated with a loss of GnRH neurons in mouse. PLoS Biol. 2014;12:e1001952.

27. McMichael G, Bainbridge $M$, Haan $E$, et al. Whole-exome sequencing points to considerable genetic heterogeneity of cerebral palsy. Mol Psychiatry. 2015;20:176

28. Wang Z-Y, Zhang H-Y. Rational drug repositioning by medical genetics. Nat Biotechnol. 2013;31:1080.

29. Brinkman RR, Dubé M-P, Rouleau GA, Orr AC, Samuels ME. Human monogenic disorders-a source of novel drug targets. Nat Rev Genet. 2006; 7:249

30. Sobreira N, Schiettecatte F, Valle D, Hamosh A. GeneMatcher: matching tool for connecting investigators with an interest in the same gene. Hum Mutat. 2015;36:928-30.

31. Strande NT, Riggs ER, Buchanan AH, et al. Evaluating the clinical validity of gene-disease associations: an evidence-based framework developed by the clinical genome resource. Am J Hum Genet. 2017;100:895-906.

32. Alazami AM,Hijazi H,Al-Dosari MS, et al. Mutation in ADAT3, encoding adenosine deaminase acting on transfer RNA, causes intellectual disability and strabismus. J Med Genet. 2013;50:425-30.

33. El-Hattab AW, Saleh MA, Hashem A, et al. ADAT3-related intellectua disability: further delineation of the phenotype. Am J Med Genet A. 2016;170:1142-7

34. Abouelhoda M, Sobahy T, El-Kalioby $M$, et al. Clinical genomics can facilitate countrywide estimation of autosomal recessive disease burden. Genet Med. 2016;18:1244.
35. Alkuraya FS. Genetics and genomic medicine in Saudi Arabia. Mol Genet Genomic Med. 2014;2:369-78.

36. Shaheen R, Alshail E, Alaqeel A, Ansari S, Hindieh F, Alkuraya FS. $T$ (brachyury) is linked to a Mendelian form of neural tube defects in humans. Hum Genet. 2015;134:1139-41.

37. Patel N, Faqeih $E$, Anazi $S$, et al. A novel APC mutation defines a second locus for Cenani-Lenz syndrome. J Med Genet. 2015;52: 317-21.

38. Shamseldin HE, Elfaki M, Alkuraya FS. Exome sequencing reveals a novel Fanconi group defined by XRCC2 mutation. J Med Genet. 2012;49: 184-6.

39. Shamseldin HE, Maddirevula S, Nabil A, Al-Fadhil S, Al Tala S, Alkuraya FS. Joint laxity in homozygotes for severe POU1F1 mutations. Am J Med Genet A. 2016;170:3356-8.

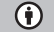

Open Access This article is licensed under a Creative Commons Attribution 4.0 International License, which permits use, sharing, adaptation, distribution and reproduction in any medium or format, as long as you give appropriate credit to the original author(s) and the source, provide a link to the Creative Commons license, and indicate if changes were made. The images or other third party material in this article are included in the article's Creative Commons license, unless indicated otherwise in a credit line to the material. If material is not included in the article's Creative Commons license and your intended use is not permitted by statutory regulation or exceeds the permitted use, you will need to obtain permission directly from the copyright holder. To view a copy of this license, visit http://creativecommons.org/licenses/ by/4.0/.

(C) The Author(s) 2018

${ }^{1}$ Department of Genetics, King Faisal Specialist Hospital and Research Center, Riyadh, Saudi Arabia. ${ }^{2}$ Department of Medical Genetics, King Faisal Specialist Hospital and Research Center, Riyadh, Saudi Arabia. ${ }^{3}$ College of Medicine, Alfaisal University, Riyadh, Saudi Arabia. ${ }^{4}$ Department of Neurosciences, King Faisal Specialist Hospital and Research Center, Riyadh, Saudi Arabia. ${ }^{5}$ Department of Pediatrics, King Faisal Specialist hospital and Research Center, Jeddah, Saudi Arabia. ${ }^{6}$ Department of Pediatrics, Prince Sultan Military Medical City, Riyadh, Saudi Arabia. ${ }^{7}$ Genetic Unit, Children's Hospital, King Saud Medical City, Riyadh, Saudi Arabia. ${ }^{8}$ Department of Pediatrics, Children's Hospital, Ain Shams University, Cairo, Egypt. ${ }^{9}$ Department of Pediatrics, Dr. Suliman Al Habib Medical Group, Riyadh, Saudi Arabia. ${ }^{10}$ Department of Pediatrics, College of Medicine, Imam Abdulrahman Bin Faisal University, Dammam, Saudi Arabia. ${ }^{11}$ Section of Clinical and Metabolic Genetics, Department of Pediatrics, Hamad Medical Corporation, Doha, Doha, Qatar. ${ }^{12}$ Genetics Division, Department of Pediatrics, King Saud Bin Abdulaziz University for Health Sciences, King Abdulaziz Medical City, Riyadh, Saudi Arabia. ${ }^{13}$ Department of Pediatrics, Security Forces Hospital, Riyadh, Saudi Arabia. ${ }^{14}$ Department of Pediatrics, King Faisal Specialist Hospital and Research Center, Riyadh, Saudi Arabia. ${ }^{15}$ Department of Obstetrics and Gynecology, King Faisal Specialist Hospital and Research Center, Riyadh, Saudi Arabia. ${ }^{16}$ Department of Neuroscience, King Faisal Specialist Hospital and Research Center, Riyadh, Saudi Arabia. ${ }^{17}$ Department of Pediatrics, Armed Forces Hospital SR, Khamis Mushayt, Saudi Arabia. ${ }^{18}$ Department of Pediatrics, College of Medicine, King Saud University, Riyadh, Saudi Arabia. ${ }^{19}$ Department of pediatrics, King Abdulaziz Medical City, Jeddah, Saudi Arabia. ${ }^{20}$ Saudi Human Genome Program, King Abdulaziz City for Science and Technology, Riyadh, Saudi Arabia. ${ }^{21}$ Medical Genetic Division, Department of Pediatrics, King Abdullah International Medical Research Centre, King Saud bin Abdulaziz University for Health Sciences, King Abdulaziz Medical City, Riyadh, Saudi Arabia. ${ }^{22}$ Department of Surgery, King Faisal Specialist Hospital and Research Center, Riyadh, Saudi Arabia 